\title{
O.S.P.
}

L'orientation scolaire et professionnelle

$36 / 1$ | 2007

Insertion, biographisation, éducation

\section{Biographisation des parcours entre projet de soi et cadrage institutionnel}

Biographisation of the courses between self-project and institutional framing

\section{Christine Delory-Momberger}

\section{(2) OpenEdition}

\section{Journals}

Édition électronique

URL : https://journals.openedition.org/osp/1243

DOI : $10.4000 /$ osp. 1243

ISSN : 2104-3795

Éditeur

Institut national d'étude du travail et d'orientation professionnelle (INETOP)

\section{Édition imprimée}

Date de publication : 15 mars 2007

Pagination : 9-17

ISSN : 0249-6739

\section{Référence électronique}

Christine Delory-Momberger, « Biographisation des parcours entre projet de soi et cadrage institutionnel », L'orientation scolaire et professionnelle [En ligne], 36/1 | 2007, mis en ligne le 05 mars 2010, consulté le 18 janvier 2022. URL : http://journals.openedition.org/osp/1243 ; DOI : https:// doi.org/10.4000/osp.1243

Ce document a été généré automatiquement le 18 janvier 2022.

(C) Tous droits réservés 


\title{
Biographisation des parcours entre projet de soi et cadrage institutionnel
}

Biographisation of the courses between self-project and institutional framing

\author{
Christine Delory-Momberger
}

\section{Introduction}

1 Les dispositifs et les modalités d'action qui doivent répondre à la mise en œuvre sur le terrain de politiques d'insertion définies à un niveau étatique sont confrontés à des logiques contradictoires: celle des parcours individuels qui inscrivent chaque bénéficiaire de mesures d'insertion dans une histoire et dans un projet singuliers, celle des cadrages institutionnels qui définissent des démarches et des programmes conçus pour des cohortes de populations en fonction de contraintes économiques et de l'état du marché de l'emploi. À cette contradiction première s'ajoute un second paradoxe touchant les exigences - peu compatibles entre elles mais souvent portées par le même discours - de la «responsabilisation" individuelle et de la gestion de plus en plus « comptable» des politiques d'insertion, soumettant l'action collective, y compris sur les terrains «qualitatifs" du social, de la santé ou de la formation, à des critères quantitatifs et productifs.

2 Ce contexte politique et économique amène à s'interroger sur les capacités des dispositifs d'insertion sociale et professionnelle à prendre en compte les singularités et les dynamiques d'élaboration des projets professionnels individuels et du travail de (re)construction de soi qu'elles impliquent. La biographisation des parcours de personnes aux prises avec des précarités sociales et professionnelles est marquée par des questionnements, des incertitudes, des fragilités, et l'émergence d'un projet professionnel durable-répondant à un projet de soi-réclamerait un espace d'exploration rendu problématique tant par l'urgence des situations individuelles que par les exigences de rentabilité collective. Comment peuvent en effet se rencontrer les 
projets personnels - qui impliquent réflexivité et maturation - et les programmes de (ré)insertion établis en fonction de conjonctures socio-économiques immédiates et fluctuantes? Quelle biographisation des parcours est générée dans la tension entre un projet de soi, souvent incertain et qui a besoin de durée et de déploiement, et les intimations du management de soi portées par un ordre économique caractérisé par la vitesse, l'hyperactivité, la rentabilité ?

Pour préciser le cadre économique et social de ce questionnement et mieux en situer les enjeux, un rapide état des lieux est nécessaire.

\section{La montée des précarités dans l'économie mondialisée}

Dans le contexte de la «nouvelle économie-monde » qui caractérise la société postindustrielle (Cohen, 2006), la question économique et la question sociale se sont dissociées et ont perdu l'équilibre qu'elles avaient réussi à trouver dans la société industrielle de l'après Seconde Guerre mondiale. La protection du travail (emploi et salaires) et la productivité économique ne sont plus dans la relation de solidarité organique qu'elles avaient connue dans le modèle fordiste qui les adossait l'une à l'autre et qui reposait sur l'intégration de tous - schématiquement les ouvriers, les ingénieurs, les patrons - au sein d'une construction verticale de l'organisation industrielle et de la hiérarchie professionnelle et sociale. Dans le contexte structurel de plein-emploi vécu par les pays occidentaux jusqu'à la fin de la période des Trente Glorieuses, le rôle de l'État consistait à apporter les protections nécessaires pour répondre à un chômage qui restait de transition et à garantir des modalités (formations, stages) de retour à l'emploi. Les ruptures qui donnent naissance à la société post-industrielle telles que les décrit Cohen - révolution technologique de l'informatique et d'internet, nouvelles fonctions $\mathrm{du}$ travail et nouvelles formes d'organisation du travail, indépendance retrouvée des marchés financiers, mondialisation de la production économique et des flux commerciaux - aboutissent à une déliaison structurelle de l'économique et du social qui ont tendance à s'autonomiser. Alors que l'organisation sociale du travail de la période fordiste produisait de l'emploi et du pouvoir d'achat, la «nouvelle économiemonde » produit une accentuation des inégalités sociales qui se manifeste, notamment, par de la précarité, de l'exclusion, de la misère sociale. La productivité économique, naguère associée à l'idée d'une amélioration générale des conditions de vie, entraîne depuis trois décennies une mise à la marge d'une population de plus en plus nombreuse. Ce "processus de refoulement hors de la sphère productive" (Paugam, 1996, p. 8) touche particulièrement les personnes les moins qualifiées mais également les jeunes, qui accèdent de plus en plus difficilement et tardivement à l'emploi, et les adultes qui, contraints au chômage par des disparitions ou des restructurations d'entreprises ou par des disqualifications de leur ancien emploi, ne retrouvent pas de travail en raison de leur âge ou parce qu'ils s'orientent difficilement vers de nouvelles professions. À cela s'ajoute la discrimination sociale et ethnique qui écarte du marché du travail une population potentiellement compétente, mais stigmatisée par ses signes " visibles ». Le nouvel ordre économique, plus soucieux de rentabilité financière que de réinvestissement productif et d'équilibre social, creuse les différences entre les individus, entre les groupes sociaux, et rejette à la périphérie les parties de la population qui ne se sont pas en mesure de s'adapter à ses exigences. 
5 Les politiques économiques et financières échappent en grande partie aux États nationaux qui peinent à ajuster leur action aux réseaux mondialisés de productivité et d'échanges. Ayant largement perdu leur capacité d'initiative, d'infléchissement et de contrôle dans le domaine économique, les États ne sont plus en mesure de protéger de manière effective la totalité de leurs membres contre des précarités qui s'installent dans la durée et contre un chômage de masse affectant depuis vingt ans un dixième environ de la population et devenu pour beaucoup un chômage d'exclusion. Maurin (2006, p. 21) note que le risque annuel de perte d'emploi s'est accru en vingt ans d'environ $30 \%$ dans tous les métiers salariés. La montée des précarités (Paugam, 2000) gagne les modalités de l'emploi qui a vu se multiplier en quelques années les formes atypiques qui sortent des définitions et des protections classiques de la condition salariale: emplois à durée limitée (CDD), missions intérimaires, emplois-jeunes, contrats professionnalisants, etc. Les enquêtes du Conseil de l'emploi, des revenus et de la cohésion sociale (CERC) (2005) montrent que l'emploi précaire et le sous-emploi, qui représentaient $16 \%$ de l'emploi total en 1990, en constituent le quart en 2000 et s'élèvent à $43 \%$ pour les non-qualifiés. On compte aujourd'hui que plus de $74 \%$ des nouveaux contrats de travail passés dans l'année se font sous une de ces formes atypiques. Au point que Castel (2007) peut parler de précariat pour désigner « cette condition sous laquelle la précarité devient un registre propre de l'organisation du travail» (p. 422).

\section{Les politiques de l'insertion entre surveillance budgétaire et responsabilisation individuelle}

6 Les politiques sociales d'insertion professionnelle - déterminées au niveau de l'État et mises en œuvre par les collectivités territoriales - tentent d'ajuster et d'adapter leurs dispositifs à des fonctionnements et à des évolutions économiques qui échappent en grande partie aux ressorts étatiques. Le travail social tel qu'il a pu se concevoir sur la base d'une intégration de chacun dans une société stable où tout le monde aurait sa place a fait long feu. Pour une population toujours plus nombreuse, passant de stage en stage et de formation en formation, l'insertion n'est plus un préalable pour accéder au marché du travail ou une étape transitoire entre deux emplois, mais un état dans lequel on s'installe durablement et qui est en passe de valoir statut social. Quant aux structures d'insertion, elles se voient de plus en plus réduites à se faire les gestionnaires de ces transits et de ces flux. "À mesure que l'accumulation des dispositifs révèle ses limites et ses effets incertains, observe Lallement (2007), les travailleurs sociaux sont obligés de basculer du travail social vers la gestion du nontravail » (p. 340).

7 Pour répondre aux défis qu'elle lui pose - précarité de l'emploi, chômage de masse, sortie du monde du travail-l'action publique est tentée d'emprunter à l'économie libérale deux principes caractéristiques de ses modes de gestion modernes. Le premier, importé des techniques d'évaluation managériales, soumet toute action, tout programme à des critères quantitatifs de productivité et de rentabilité. En France, la récente mise en application de la loi organique relative aux lois de finances (LOLF) répond à cette "économicisation" de la gestion publique et vise à réduire et à rationaliser la dépense de l'État. Son principe est de soumettre la budgétisation des crédits aux résultats obtenus par les programmes mis en place et, dans des domaines 
comme celui de l'insertion, d'appliquer des critères de rentabilité comptables et économiques à des facteurs, des procédures, des objectifs essentiellement " humains ». Ces conditions administratives et budgétaires complexifient considérablement la tâche du travail social qui se trouve aujourd'hui dans une situation paradoxale. Il lui faut en effet à la fois remplir son rôle politique d'intégrateur social dans une société instable prise dans l'hypervitesse de ses adaptations successives à l'ordre économique mondial et tenter d'élaborer avec le "client" un parcours qui voudrait cibler une insertion durable. La surveillance étatique des programmes par des opérations quantifiées de contrôle élaborées dans le contexte de la LOLF incite à s'interroger sur leurs conditions de réalisation et de succès. Leur réussite supposerait que le face-à-face entre le professionnel du travail social et l'usager se fasse sur le double fondement-bien difficile à concilier et à établir-d'une rationalité évaluative commandée par l'institution et de l'ouverture à un projet professionnel de la part de personnes dont les précarités économiques, sociales, psychologiques n'auraient pas entamé les capacités d'affirmation de soi.

8 Le second principe relève à la fois d'un discours idéologique - qui se pare volontiers de valeurs éthiques - et d'une pratique qui s'inscrit dans des dispositifs législatifs et des mesures techniques. Le contexte discursif et idéologique est celui d'une "société des individus » dans laquelle chacun est sommé d'affirmer sa subjectivité et sa singularité, d'être l'auteur et l'acteur de sa vie, de développer un projet de vie, en se fixant à soimême ses principes d'action et d'évaluation (Delory-Momberger, 2003, pp. 49-52) et en trouvant des solutions biographiques aux contradictions sociétales (Beck, 2001). Transposée dans le monde du travail et de l'entreprise, cette intimation collective à " être soi » se transforme en injonction au management de soi : il revient à chacun d'être le meilleur et le plus performant, de se faire "l'entrepreneur" de soi-même (Ehrenberg, 1991) ou, comme le dit encore Beck développant la formule allemande du «Ich-AG» (littéralement et significativement : «entreprise-moi »), d'être "le bureau d'étude de son propre curriculum». Ce discours ne va pas sans ambiguïté ni contradiction et sert bien souvent à occulter la déresponsabilisation des entreprises et de la collectivité, en particulier à l'endroit des catégories de travailleurs les moins favorisés. Comme le font remarquer Grosjean et Sarnin (2002), « le report quasi exclusif sur l'individu de la gestion de son parcours (...) peut apparaître comme un progrès pour ceux qui ont des marges de choix dans leur système d'activités, et comme une régression sociale, doublée d'une épreuve psychologique chez les travailleurs précaires, puisque les individus sont mis dans la situation paradoxale de considérer que leur parcours dépend d'eux alors que ce qu'ils vivent est un parcours subi. (...) Il en est de même chez les ouvriers où le discours sur la gestion individuelle du parcours vient heurter de plein fouet l'absence de projection possible dans une carrière » (p. 16).

9 Ce discours du management de soi trouve son point d'accroche dans la notion d'employabilité entendue comme capacité de l'individu à mobiliser ses ressources et ses compétences pour convaincre de son adéquation aux conditions d'emploi qui lui sont proposées ou pour susciter l'offre à laquelle il postulerait de façon idéale. À la «valeur» employabilité sont étroitement associées d'autres "qualités» qui sont requises par «le nouvel esprit du capitalisme» (Boltansky \& Chiapello, 1999) et qui constituent la force de vente du demandeur d'emploi entrepreneur de soi-même: capacité à adapter et à infléchir son action en fonction de conditions nouvelles (flexibilité), disponibilité géographique et horaire (mobilité), distance réflexive et évaluative par rapport à soi-même et à son action (réflexivité/self evaluation), capacité à 
remettre sur le chantier une formation et des compétences jamais définitivement acquises (apprentissage tout au long de la vie/life long learning), capacité - et la boucle est bouclée - à assurer sa propre promotion sur le marché du travail (bench marking). Cette idéologie, qui tend à rechercher chez les seuls individus les raisons de leur précarité sociale et professionnelle et les ressources de leur accès à l'emploi, a largement gagné le terrain du travail social et de l'insertion et a contribué à en changer le sens en transformant la nature éthique du rapport social au travail: "L'insertion, écrivent Lebaron et Schultheis (2007), qui fut longtemps pensée comme un ensemble de droits associés au risque du chômage et à l'entrée sur le marché du travail, est désormais conçue comme un ensemble de "devoirs" particuliers des demandeurs d'emploi et des inactifs pour devenir des salariés efficaces, y compris dans la recherche d'emploi» (p. 880).

10 C'est dans ce contexte, qui allie précarité de l'emploi, rationalisation budgétaire et responsabilisation individuelle, qu'il faut comprendre les mesures d'incitation à l'emploi adoptées dans plusieurs pays européens et inspirées du workfare américain ${ }^{1}$ : il s'agit de contraindre les chômeurs et les inactifs, par une incitation morale et juridique qui peut être liée à une contrepartie matérielle (du type du récent RMA/revenu minimum d'activité), d'accepter des emplois qui sortent de normes de travail et de conditions de rémunération clairement référencées. Cette politique d'«activation »a un caractère nettement contraignant et a pour objet, en particulier lorsqu'elle s'adresse à des jeunes en recherche de premier emploi, de créer des acteurs économiques mobiles et flexibles capables de développer des stratégies pour lutter contre les risques liés au chômage et s'assurer une "activité » éventuellement très éloignée d'un emploi au sens reconnu de ce terme. "On assiste, écrit Castel (2007), à un glissement de l'emploi classique vers des formes d'activités en deçà de l'emploi et qui pourrait déboucher à la limite sur une société de pleine activité toute différente d'une société de plein-emploi » (p. 417).

\section{Biographie de travail et processus de biographisation}

11 Les conditions du travail et de l'emploi dans les sociétés post-industrielles, les mutations qui brouillent les frontières autrefois clairement délimitées de l'emploi et du non-emploi, le développement du travail précaire et de formes d'emploi ou d'activité atypiques, la prégnance du chômage de masse et l'exclusion de la sphère du travail qu'il représente pour des millions de personnes sont autant de réalités économiques et sociales appelées à transformer en profondeur les biographies de travail et les opérations de biographisation qui leur sont liées. Rappelons que nous entendons par biographie, non le cours réel des existences dans leur déroulement factuel, mais l'ensemble des représentations selon lesquelles les individus se figurent leur vie et en construisent continûment la forme (les formes) et le sens (les sens), et par biographisation le processus incessant par lequel ils produisent pour eux-mêmes et pour les autres les manifestations mentales, mais aussi verbales, corporelles, comportementales de leur existence (Delory-Momberger, 2004b). La figuration narrative - la vie comme histoire et comme récit-représente une des dimensions privilégiées (et en quelque sorte étymologiques) de la biographie, mais l'activité biographique peut prendre de multiples formes qui ne relèvent pas exclusivement du langage verbal. 
12 Les constructions biographiques individuelles sont étroitement dépendantes des contextes socio-historiques et culturels et des modèles et programmations biographiques dont ils sont porteurs (Delory-Momberger, 2005). Les différents secteurs de l'existence et les milieux sociaux dans lesquels se déroule l'activité humaine sont susceptibles de générer des représentations biographiques spécifiques qui se concrétisent sous forme de récits idéaux-typiques qui servent de conducteurs aux biographies individuelles. Ces patrons biographiques évoluent au fur et à mesure des transformations sociétales, mais leur prégnance dans les représentations collectives et individuelles peut provoquer des effets de décalage qui amènent par exemple à ce que se prolongent au sein d'une collectivité des modèles qui appartiennent à un état de société antérieur et qui ne sont plus en phase avec les mutations et les évolutions en cours.

13 C'est sans doute à un de ces moments de déphasage que nous assistons aujourd'hui dans les biographies de travail: le modèle sur lequel les «travaillants" continuent à « vivre » - ou plutôt à se biographier, c'est-à-dire à se représenter leur histoire de travail est celui d'une trajectoire continue, linéaire, même si, pour la plupart d'entre eux, ils ne peuvent plus dans les faits raconter une telle histoire ${ }^{2}$. La matrice reste celle de la carrière, d'un parcours qui suit les âges de la vie, débute par des phases d'apprentissage et de formation, se développe selon une pente ascensionnelle et s'achève par un retrait (une retraite) vécu comme une récompense du labeur enduré. Cette image (cet imaginaire) du métier ou de la profession, héritée de l'âge industriel, a beau n'être (presque) plus jamais réalisée, elle est celle qui continue à informer les représentations de la vie au travail. Cette figure d'une existence de travail, qui véhicule avec elle tout un monde de vie (représentations, valeurs, croyances, images symboliques) lié à un état antérieur de l'organisation économique et sociale, entre ainsi en contradiction, non seulement avec les mutations de l'emploi (et du non-emploi) et avec les bouleversements du cycle de vie qu'elles provoquent dans les économies postindustrielles, mais avec les injonctions du management de soi et les «valeurs » qu'elles promeuvent de flexibilité, de mobilité, d'employabilité. Dès lors, les processus de biographisation sont dans une sorte de porte-à-faux ou de fuite en avant : les expériences de travail ne trouvant plus directement à s'ancrer dans des représentations biographiques qui les intègrent et leur fournissent des modalités de forme et de sens, les individus sont amenés ou bien à une relative confusion et dissolution dans la manière dont ils biographient ces expériences ou bien au contraire à un surinvestissement forcément déceptif de schémas biographiques anciens. Dans le premier cas, l'absence de repères et de représentations intégratives peut conduire à des formes de déshérence et de solitude socio-biographique, dans le second la tentative vainement poursuivie de faire coïncider des expériences de travail marquées par la fragmentation et la discontinuité avec la représentation valorisée d'une trajectoire professionnelle linéaire et orientée suscite amertume et défiance de soi. C'est à de telles difficultés " biographiques » que sont exposés au premier chef les " usagers ", jeunes et adultes, de l'insertion, lorsqu'ils sont confrontés aux dispositifs qui doivent leur permettre d'accéder ou de revenir à l'emploi. 


\section{Scénarios biographiques et « technologies de soi »}

\section{l'emploi de plus en plus instables et flexibles, les politiques sociales d'insertion sont} amenées à définir des profils et des trajectoires professionnels marqués eux aussi par la réactivité et la flexibilité. Pour les personnes en situation d'insertion professionnelle, ces parcours institués prennent la forme de "scénarios biographiques " préétablis auxquels elles doivent confronter leur propre biographie individuelle et professionnelle (Delory-Momberger, 2006).

Parmi les dispositifs mis en place au titre des politiques d'insertion, les accompagnements de projet professionnel mettent en œuvre des « technologies de soi » qui ne relèvent plus des classiques techniques de soi telles que Foucault (1984) a pu les décrire ${ }^{3}$, mais qui se présentent comme des techniques de réactivité et d'ajustement des publics ciblés à un environnement socio-économique qui fait de l'adaptabilité, de la flexibilité et de la réactivité les moteurs d'une réussite conçue en termes exclusifs de rentabilité et de profit et qui projette ces «valeurs" sur ceux qui doivent être les " agents» de cette réussite et qui le seront d'autant mieux qu'ils les "incarneront » dans leurs comportements et leur existence. Ces dispositifs conduisent à problématiser la figure d'un «sujet» capable d'assurer par lui-même la gestion de son «capital biographique » et à s'interroger sur la possibilité, en particulier pour les personnes en situation de précarité, de "se construire" en réponse aux réquisits de cette biotechnologie de l'insertion. Soumise à l'urgence de l'immédiateté et du comportement-réflexe, cette biotechnologie est en effet en complète opposition avec la latence et la maturation que réclame un mouvement de retour sur soi (de réflexivité, et non de réflexe), elle ne vise pas à engager la personne dans un travail biographique qui lui permette de se construire et de trouver la place qui est « la sienne »; elle a au contraire de plus en plus pour objectif d'ajuster les existences individuelles et les biographies professionnelles aux conditions des flux du marché et de l'emploi d'une part, de la gestion comptable des politiques sociales d'autre part. Aussi les scénarios qu'elle propose sous forme de parcours ou d'accompagnements dits personnalisés ne peuventils plus correspondre à des projets d'existence qu'il serait possible d'investir biographiquement, mais répondent à des programmes, à des objectifs auxquels on peut au mieux « prêter » sa vie pour précisément « survivre ».

\section{BIBLIOGRAPHIE}

Beck, U. (2001). La Société du risque. Paris : Aubier.

Boltanski, L. \& Chiapello, E. (1999). Le Nouvel esprit du capitalisme. Paris : Gallimard.

Boyer, R. (2007). Comment concilier solidarité sociale et efficacité économique à l'ère de la globalisation : une lecture régulationniste. In S. Paugam (éd.), Repenser la solidarité. L'apport des sciences sociales (pp. 887-914). Paris : Presses universitaires de France.

L'orientation scolaire et professionnelle, 36/1 | 2007 
Castel, R. (2007). Au-delà du salariat ou en deçà de l'emploi ? L'institutionnalisation du précariat. In S. Paugam (éd.), Repenser la solidarité. L'apport des sciences sociales (pp. 415-433). Paris : Presses universitaires de France.

Cohen, D. (2006). Trois leçons sur la société post-industrielle. Paris : Seuil.

Conseil de l'emploi, des revenus et de la cohésion sociale (CERC) (2005). La Sécurité de l'emploi face au défi des transformations économiques (Rapport $\mathrm{n}^{\circ}$ 5). Paris : La Documentation française.

Delory-Momberger, C. (2003). Biographie et éducation. Figures de l'individu-projet. Paris : Anthropos. Delory-Momberger, C. (2004a). Les Histoires de vie. De l'invention de soi au projet de formation ( $1^{\mathrm{re}}$ éd. 2000). Paris : Anthropos.

Delory-Momberger, C. (2004b). Biographie, socialisation, formation. Comment les individus deviennent-ils des individus? L'Orientation scolaire et professionnelle, 33 : Travail biographique, construction de soi et formation (coord. C. Delory-Momberger), 551-570.

Delory-Momberger, C. (2005). Histoire de vie et recherche biographique en éducation. Paris : Anthropos.

Delory-Momberger, C. (2006). Scénarios biographiques et « technologies de soi » dans les politiques sociales d'insertion professionnelle. In J. Biarnès \& C. Delory-Momberger (éd.), L'Acteur social : le sujet et l'évaluation des politiques sociales (pp. 117-126). Nantes : Éditions Pleins Feux.

Ehrenberg, A. (1991). Le Culte de la performance. Paris : Hachette.

Foucault, M. (1984). Dits et écrits IV. Paris : Gallimard.

Grosjean, M. \& Sarnin, P. (2002). Les Parcours professionnels. Éducation permanente, 150 (1), 9-22.

Lallement, M. (2007). Le Travail. Une sociologie contemporaine. Paris : Gallimard.

Lebaron, F. \& Schultheis, F. (2007). Vers un État social européen ? Les enseignements de la politique européenne de lutte contre le chômage des jeunes (1997-2001). In S. Paugam (éd.), Repenser la solidarité. L'apport des sciences sociales (pp. 873-886). Paris : Presses universitaires de France.

Maurin, E. (2006). Les Nouvelles précarités. In La Nouvelle critique sociale (pp. 19-25). Paris : Seuil.

Paugam, S. (éd.) (1996). L'Exclusion. L'état des savoirs. Paris : La Découverte.

Paugam, S. (2000). Le Salarié de la précarité. Paris : Presses universitaires de France.

Paugam, S. (éd.) (2007). Repenser la solidarité. L'apport des sciences sociales. Paris : Presses universitaires de France.

Sennett, R. (2000). Le Travail sans qualités. Les conséquences humaines de la flexibilité. Paris : Albin Michel.

\section{NOTES}

1. «Le workfare s'entend comme une stratégie visant, pour l'essentiel, à réduire la part des budgets sociaux grâce à une baisse générale des normes antérieures et à l'institution d'une série d'incitations faisant sentir aux salariés la pression à l'acceptation d'un emploi quel qu'il soit. Fondamentalement, les gouvernements comptent sur le renforcement du rôle des marchés pour que les individus exercent des choix les plus favorables à l'emploi, dans un contexte où les indemnités de chômage sont réduites et limitées dans le temps. En ce sens, il s'agit d'une 
remarchandisation de la relation salariale, dès lors que l'individu ne peut plus compter sur le soutien d'un revenu assuré indépendamment de l'exercice d'une activité » (Boyer, 2007, p. 904).

2. Le sociologue américain Sennett (2000) rapporte qu'au début des années 1970 les ouvriers et les employés racontaient encore l'histoire de leur vie selon une structure linéaire continue et orientée, dont l'activité professionnelle (usine, bureau) constituait le motif organisateur et intégrateur : cette histoire était la leur et ils s'identifiaient à elle. Vingt années plus tard, la capacité de produire un récit unifié de la vie s'est considérablement réduite : à la place d'une histoire unique qui intègre tous les aspects de la vie sous un schème social et professionnel dominant, les informateurs délivrent des histoires plurielles et éclatées entre lesquelles ils peinent à établir de la continuité et de la permanence.

3. Dans la description archéologique des moyens par lesquels une société aménage son vécu collectif, Foucault (1984), à côté des techniques de production, des techniques symboliques et des techniques de pouvoir, reconnaît l'existence de techniques de soi, qu'il définit comme des techniques qui «permettent aux individus d'effectuer, seuls ou avec d'autres, un certain nombre d'opérations sur leur corps et leur âme, leurs pensées, leurs conduites, leur mode d'être » (p. 785).

\section{RÉSUMÉS}

La mondialisation économique et technologique a transformé les conditions du travail et a introduit des facteurs d'instabilité et de précarité qui se traduisent par un accès plus difficile à l'emploi et par un chômage de masse. Pour répondre à ces nouveaux défis, les politiques d'insertion professionnelle, alors que pèse sur elles un contrôle accru de leurs pratiques et de leurs résultats, doivent à la fois « encadrer » institutionnellement des populations de plus en plus nombreuses et «accompagner» individuellement des parcours singuliers. On s'interroge dans cet article sur la capacité des dispositifs d'insertion à mettre en place les conditions qui permettraient l'élaboration de véritables projets personnels et on souligne les contradictions entre techniques de management de soi et processus de construction de soi.

Economic and technical internationalisation has transformed working conditions and has introduced factors of instability and precariousness that are translated by a poorer job accessibility is concerned and mass unemployment. In order to respond to these new challenges, in spite of increasing external control of procedures and their results, policies of professional integration must both "manage" institutionally an ever-growing population and "accompany" individually singular trajectories. In this article, we question the capacity of current measures of integration to implement the necessary conditions for the elaboration of genuine personalized projects and we underline the contradictions between techniques of self-management and the processes of self-construction. 
INDEX

Mots-clés : Biographie, Biographisation, Emploi, Management de soi, Politiques d'insertion, Précarité, Projet de soi, Responsabilisation individuelle, Technologies de soi

Keywords : Biographisation, Biography, Employment, Individual responsibility, Integration policies, Precariousness, Self-management, Self-project, Self-technologies

\section{AUTEUR}

\section{CHRISTINE DELORY-MOMBERGER}

Christine Delory-Momberger est professeur en sciences de l'éducation à l'université Paris 13/ Nord. Elle est membre du Centre de recherche interuniversitaire EXPERICE (Expérience, ressources culturelles, éducation), à Paris 13/Nord (avenue Jean-Baptiste Clément, 93430

Villetaneuse) - Paris 8/Saint-Denis, et responsable d'un master professionnel « Ingénierie des programmes d'insertion ». Courriel : delbourg@club-internet.fr. 